\title{
Effect of Indomethacin on the Regulation of Cerebral Blood Flow during Respiratory Alkalosis in Newborn Piglets
}

\author{
PATRICIA A. DeGIULIO, RUSSELL A. ROTH, OM P. MISHRA, \\ MARIA DELIVORIA-PAPADOPOULOS, AND L. CRAIG WAGERLE \\ Depariment of Physiology, University of Pennsylvania School of Medicine, Philadelphia, Pennsylvania 19104
}

\begin{abstract}
The effect of cyclooxygenase inhibition by indomethacin on regional cerebral blood flow (CBF) during hypocapnia induced by hyperventilation and during hypercapnia induced by $\mathrm{CO}_{2}$ inhalation was examined. $\mathrm{CBF}$ was measured in 27 anesthetized, ventilated piglets (2-8 d) using microspheres in control and indomethacin treated animals $(5 \mathrm{mg} / \mathrm{kg})$ after hyperventilation or inhalation of $6 \% \mathrm{CO}_{2}$. In the control group $(n=6)$, CBF decreased significantly $(p<0.05)$ to all regions of the brain after hyperventilation with a $32 \%$ decrease in the cerebral cortex. In the indomethacin-treated group ( $n=6$ ), blood flow significantly decreased by 35 to $49 \%$ in all regions of the brain, except the cerebral white matter, during normocapnia with no further decrease in flow during subsequent hypocapnia. Although CBF increased significantly after indomethacin treatment during hypercapnia the response was markedly attenuated with blood flow to the cerebral gray matter, hippocampus and pons rising onfy 42,25 , and $42 \%$ in contrast to 108,75 , and $225 \%$ in the control group. Since indomethacin decreased resting $\mathrm{CBF}$, unilateral sympathetic nerve stimulation at $15 \mathrm{~Hz}$ was used to test the specificity of indomethacin on hypocapnic vasoconstriction $(n=5)$. Unilateral sympathetic nerve stimulation caused a further statistically significant decrease in CBF on the stimulated side after hyperventilation with indomethacin $(12 \%)$, which was comparable to that which occurred during normocapnia (16\%). The data demonstrate that indomethacin attenuates the cerebrovascular sensitivity to both increases and decreases in $\mathrm{CO}_{2} / \mathrm{H}^{+}$and implicate a possible role for vasoactive prostanoids in mediating the response of CBF to fluctuations in $\mathrm{CO}_{2}$ in newborn piglets. (Pediatr Res 26: 593-597, 1989)
\end{abstract}

\section{Abbreviations}

CBF, cerebral blood flow

USS, unilateral sympathetic stimulation

CSF, cerebrospinal fluid

MABP, systemic mean arterial blood pressure

Current evidence supports a role for prostaglandins in the regulation of CBF during hypercapnia $(1,2)$, seizures (3), and asphyxia (4) in the newborn piglet. Each of these conditions is

Received April 21, 1989; accepted July 24, 1989.

Correspondence Patricia A. DeGiulio M.D., c/o L. Craig Wagerle, Ph.D., Departnent of Pliysiology, University of Pennsylvania School of Medicine, B405 Richards Bldg., Philadelphia, PA 19104.

Supported by grants from the NIH, National Heart, Lung, and Blood Institute (RO1/HL-37421), National Institute of Neurological and Communicative Disorders and Stroke (R29/NS-19762) and (5T32/HL-07027). associated with cerebral acidosis and results in increased CBF (5-9). Accordingly, studies from this laboratory have shown that indomethacin and phospholipase $\mathrm{A}_{2}$ inhibitors attenuate the cerebrovascular dilation to hypercapnia as well as to changes in local $\left[\mathrm{H}^{+}\right](2)$, suggesting that prostaglandins contribute to the regulation of $\mathrm{CBF}$ in response to changes in $\mathrm{pH}$ in the newborn piglet.

Hyperventilation is commonly used in the treatment of persistent pulmonary hypertension of the newborn. However, severe hypocapnia $\left(\mathrm{PaCO}_{2}<20 \mathrm{~mm} \mathrm{Hg}\right.$ ) and local hypocapnic alkalosis have been shown to decrease CBF and to constrict the pial arterioles in several species (10-13). Inasmuch as this effect is mediated through a decrease in $\left[\mathrm{H}^{+}\right](14)$, indomethacin might alter this response by either decreasing the production of vasodilator prostanoids and thus augment the vasoconstriction, or by diminishing the response of the vascular smooth muscle to $\mathrm{H}^{+}$ and attenuate the decrease in CBF.

Our investigation examines the effect of cyclooxygenase inhibition by indomethacin on regional CBF during hypocapnia induced by hyperventilation and during hypercapnia induced by $\mathrm{CO}_{2}$ inhalation. In addition, because indomethacin causes acute cerebral vasoconstriction in the newborn piglet ( 1 ), we compared the magnitude of regional changes in $\mathrm{CBF}$ during hypocapnia to that induced by indomethacin alone. To test the specificity of the effect of indomethacin on hypocapnic vasoconstriction, sympathetic nerve stimulation was studied testing the limit of the vasoconstrictor response to hypocapnia in the presence of indomethacin.

\section{MATERIALS AND METHODS}

Animal Preparation. Studies were performed on 27 newborn Yorkshire piglets, 2-8 d of age, weighing 1.1-2.8 kg. Anesthesia was induced with $4 \%$ halothane- $96 \% \mathrm{O}_{2}$ and maintained with $0.5 \%$ halothane and a balance of $\mathrm{O}_{2}$. Lidocaine $(2 \%)$ was injected subcutaneously before incision. A tracheostomy was performed and polyethylene catheters were placed into the left femoral artery for monitoring blood pressure and blood gases, right brachial artery for withdrawal of blood during microsphere injection, and left femoral vein for administration of drugs. A continuous intravenous infusion $(12 \mathrm{~mL} / \mathrm{h}, 5 \%$ glucose $)$ was given. A 3.5 Fr polyvinyl pig-tailed catheter was inserted into the right femoral artery and passed into the left ventricle for injection of microspheres. Blood pressure and heart rate were monitored (Gould recorder with a Gould Statham P23XL pressure transducer, Gould Inc, Rolling Meadows, IL), as was temperature and end-tidal $\mathrm{CO}_{2}$ (Puritan-Bennett Datex model, Datex Medical Instrumentation Inc., Wilmington, MA).

Arterial blood samples were analyzed for $\mathrm{pH}, \mathrm{PCO}_{2}$, and $\mathrm{PO}_{2}$ using electrodes (Corning model 178, Ciba Corning Diagnostics Corp, Medfield, MA). Hb concentration and saturation were measured on a co-oximeter (Instrumentation Laboratories, Lex- 
ington, MA) and hematocrit determined by the micromethod. At least $60 \mathrm{~min}$ before experimental manipulation halothane was discontinued and the animals sedated $(0.067 \mathrm{~mL} / \mathrm{kg}$ InnovarVet, Taylor Pharmaceuticals, Decatur, IL; fentanyl $0.4 \mathrm{mg} / \mathrm{mL}$ + droperidol $20 \mathrm{mg} / \mathrm{mL})$, paralyzed $(0.5 \mathrm{mg}$ pancuronium bromide), and mechanically ventilated (Bournes BP200, Bear Medical, Riverside, CA) with $75 \%$ nitrous oxide and $25 \% \mathrm{O}_{2}$. Supplemental doses of pancuronium and Innovar-Vet were given every 30 to $60 \mathrm{~min}$. Ventilation was adjusted to maintain end-expired $\mathrm{PCO}_{2}$ at $30-35 \mathrm{~mm} \mathrm{Hg}$ and measured $\mathrm{PaO}_{2}$ maintained between $80-100 \mathrm{~mm} \mathrm{Hg}$. The animals body temperature was maintained at $38-39^{\circ} \mathrm{C}$ rectal using a warming blanket.

Measurement of $C B F$. CBF was measured by the tracer microsphere technique (15). Microspheres $(15 \mu \mathrm{m})$ labeled with ${ }^{141} \mathrm{Ce}$, ${ }^{85} \mathrm{Sr},{ }^{46} \mathrm{Sc}\left(3 \mathrm{M}\right.$, St. Paul, MN), or ${ }^{113} \mathrm{Sn}$ (New England Nuclear, Boston, MA) were used. Stock solutions were sonicated for 5 min and shaken vigorously on a vortex mixer for 2-5 min. Approximately $0.7-1.2 \times 10^{6}$ microspheres were placed into specially prepared injection vials and shaken again on a vortex mixer before injection. The contents of the injection vial were flushed into the left ventricle over a period of $15-30 \mathrm{~s}$. Reference arterial blood was withdrawn from the right brachial artery for $10 \mathrm{~s}$ before, during, and for $60 \mathrm{~s}$ after the microsphere injection at a rate of $0.97 \mathrm{~mL} / \mathrm{min}$.

At the conclusion of the experiment the animal was killed by intravenous injection of $\mathrm{KCl}$. The brain was removed and sectioned into right and left cerebrum, caudate nucleus, hippocampus, thalamus-hypothalamus, midbrain, cerebellum, pons, and medulla. In addition to the brain, the left and right choroid plexus (from the lateral ventricles), left and right masseter muscles, kidneys, and heart were also removed. Tissues were weighed and placed into counting vials along with reference bloods. Counting vials were filled to a height of less than $2.5 \mathrm{~cm}$ to minimize geometrical counting error.

The amount of radioactivity present in the tissues and reference blood samples was measured on a $\gamma$-counter and blood flow to the tissues and cardiac output were calculated as previously described $(15,16)$.

Experimental Protocols. Effect of indomethacin on CBF response to hypocapnia. In 12 piglets, the effect of hypocapnia on $\mathrm{CBF}$ was investigated with and without indomethacin (a cyclooxygenase inhibitor). In the control group $(n=6)$, baseline measurements were made during normoxic normocapnia. Minute ventilation was then increased by adjusting the rate and peak inspiratory pressure to achieve an end-expiratory $\mathrm{CO}_{2}$ concentration of $1-1.5 \%$. Thirty min were allowed to achieve stable $\mathrm{PaCO}_{2}$ and repeat measurements of CBF were made. In the indomethacin group $(n=6)$, after baseline CBF measurements sodium indomethacin trihydrate (a gift from Mr. William Henckler, Merck, Sharpe \& Dohme Research Laboratories, Rahway, NJ) diluted in normal saline, $5 \mathrm{mg} / \mathrm{kg}$, was slowly administered intravenously. This dose of indomethacin has been shown to cross the blood-brain barrier and decrease the synthesis of cortical 6-keto-prostaglandin $F_{1 \alpha}$, prostaglandin $E_{2}$, and thromboxane $B_{2}$ to one-tenth as measured in the subarachnoid CSF of newborn piglets $(1,17)$. CBF measurements were repeated 30 min after the administration of indomethacin was completed. Minute ventilation was then increased as previously described allowing 30 min for equilibration and repeat measurements of CBF during hyperventilation were then obtained. Arterial blood was sampled prior to each CBF measurement and analyzed for blood gases, $\mathrm{pH}, \%$ Hb saturation and hematocrit.

Effect of indomethacin on CBF response to hypercapnia. The effect of indomethacin on $\mathrm{CBF}$ in response to increased $\mathrm{CO}_{2}$ was examined in 10 piglets. In the control group $(n=5), \mathrm{CBF}$ was measured during baseline and again after the administration of $6 \% \mathrm{CO}_{2}$ to the inspiratory gas mixture for $20 \mathrm{~min}$. In the second group $(n=5), C B F$ was measured during baseline, $30 \mathrm{~min}$ after the administration of $5 \mathrm{mg} / \mathrm{kg}$ intravenous indomethacin, and again after 20 min of inspiring $6 \% \mathrm{CO}_{2}$ in the gas mixture.
Effect of sympathetic nerve stimulation on $C B F$. In five animals both left and right cervical sympathetic ganglia were isolated and nerve integrity confirmed by observing ipsilateral pupillary dilatation and piloerection in response to applying a brief current to the nerve. Bipolar electrodes were placed around each nerve immediately caudal to the superior cervical sympathetic ganglion and the nerve was cut proximal to the electrodes on each side. Most of the sympathetic fibers innervating the cerebral vasculature originate from the ipsilateral superior cervical sympathetic ganglion $(18,19)$. Thus, the effect of unilateral electrical stimulation of the superior cervical sympathetic ganglion on cerebral vessels is primarily on the ipsilateral hemibrain, whereas the contralateral hemibrain can serve as control when measuring CBF with microspheres. In the present experiments, the ganglion was stimulated on one side with the unstimulated side serving as control. The side to be stimulated was randomized. Stimulation parameters were $15 \mathrm{~Hz}, 20 \mathrm{~V}$, and $3 \mathrm{~ms}$. Stimulation was continued for 1-2 min and CBF measured between 45 and $90 \mathrm{~s}$ of the stimulation period. CBF was measured with unilateral sympathetic stimulation occurring at the time of microsphere injection during baseline, and again $30 \mathrm{~min}$ after hyperventilation in the presence of indomethacin.

Statistical Analysis. CBF, systemic MABP, cardiac output, heart rate, blood gases, and $\% \mathrm{Hb} \mathrm{O}$ saturation were analyzed using repeated measure analysis of variance. For significant $F$ values, the Student-Newman-Keuls test was performed. Percent differences were analyzed after arc sine transformation. The Student's $t$ test was performed on the arc sine of the change in percent. A level of $p<0.05$ was considered significant. Values are reported as means \pm SEM.

\section{RESULTS}

Effect of indomethacin on CBF response to hypocapnia. During hyperventilation arterial $\mathrm{PCO}_{2}$ decreased with pH increasing accordingly, heart rate increased and MABP fell in the control and indomethacin group by a similar magnitude (Table 1). Hematocrit remained unchanged, whereas cardiac output decreased after indomethacin administration in all groups. This change, although significant $(p<0.05$ ), would not be expected to affect CBF because mean arterial blood pressure was unchanged after indomethacin treatment (Table 1). In the control group blood flow decreased to all regions of the brain after hyperventilation with the largest $\%$ decrease in the choroid plexus $(48 \%)$ with flow decreasing significantly to the cerebral gray matter by $32 \%$. In the indomethacin group during normocapnia, blood flow decreased significantly to all regions of the brain with a $40 \%$ decrease to the cerebral gray matter. Subsequent hypocapnia did not cause a further decrease in the blood flow to any region studied except for the choroid plexus (Table 1).

Effects of indomethacin on CBF response to hypercapnia. With addition of $\mathrm{CO}_{2}$ to the inspirate, arterial $\mathrm{PCO}_{2}$ increased similarly in both groups, whereas blood pressure, heart rate, and cardiac output remained unchanged (Table 2). Administration of indomethacin decreased cardiac output without a significant change in blood pressure or heart rate. CBF increased significantly to all regions of the brain during hypercapnia with the greatest change observed in the brain stem (Table 2). During normocapnia, blood flow decreased to all regions of the brain after indomethacin was administered. The increase in CBF during hypercapnia, although significant was markedly attenuated in the presence of indomethacin. Blood flow to the cerebral gray matter, hippocampus and pons increased only 42,25 , and $42 \%$ in contrast to 108,75 , and $225 \%$ in the control group. Blood flow to the choroid plexus, however, remained unchanged during hypercapnia with or without indomethacin.

Effect of sympathetic stimulation on CBF. Sympathetic stimulation had no affect on $\mathrm{MABP}$, heart rate, $\mathrm{pH}, \mathrm{PaCO}_{2}$, or $\mathrm{PaO}_{2}$. Unilateral stimulation of the sympathetic trunk during normocapnia reduced blood flow to the ipsilateral side by $16 \pm 2 \%$ in 